\title{
Sobre las preferencias léxicas en los registros coloquial y formal en L1 y L2
}

\section{On lexical preferences in colloquial and formal registers in L1 and L2}

\author{
Raúl Fernández Jódar \\ Universidad Adam Mickiewicz de Poznań \\ raulfdez@amu.edu.pl
}

\begin{abstract}
As is well known, formal and colloquial registers should not be understood as two independent departments without any relation between them, but as two extremes of the continuum of speech. It should be added that this continuum will be different for speakers of different languages, since certain lexical, pragmatic or syntactic elements, among others, can be understood as corresponding to different registers, either due to the influence of the $\mathrm{L} 1$ or the learning process. The aim of this study is to analyze the existence of pairs of lexical preferences according to the register. In addition, it will be checked if these lexical preferences are given in both L1 and L2.
\end{abstract}

Keywords: register, colloquial, formal, lexical preferences, Spanish as a foreign language

\section{LAS PREFERENCIAS LÉXICAS}

Gaviño Rodríguez define las preferencias léxicas como aquellas "que se manifiestan en determinadas parejas de elementos tradicionalmente consideradas sinónimas pero cuyo uso difiere notablemente en función del registro en que nos expresemos" (2008: 75). Sin embargo, como es bien sabido, todas las lenguas varían a lo largo del tiempo debido a su propia manifestación como actividad. Dichas variaciones no responden a un único criterio. Así, tenemos variaciones diatópicas en referencia al espacio geográfico, variaciones diastráticas en referencia a los estratos socioculturales, variaciones diacrónicas en referencia a la lengua de una determinada 
época y variaciones diafásicas en referencia a las modalidades expresivas como estilos y registros de una lengua (Coseriu 1981). De ahí que la lengua no sea considerada como un sistema sino como un diasistema, es decir, un conjunto de sistemas.

En principio, al tratar las preferencias léxicas en los registros, parece obvio que debemos centrarnos en las variaciones diafásicas de la lengua. Sin embargo, no debemos perder de vista que considerar una lengua como un conjunto de sistemas significa que a la hora de analizar un término no podemos únicamente centrarnos en una de las posibles variaciones, sino que debemos tener en cuenta todas las posibilidades. Por ejemplo, las diferencias que pueden aparecer entre palabras como enojarse/enfadarse, plata/dinero o manejar/conducir, no se deben únicamente a variaciones diafásicas, sino que también habría que considerar otras variaciones.

En nuestra investigación nos centraremos en parejas de palabras propuestas en Gaviño Rodríguez (2008: 76), según el cual estas se dan en el español peninsular y presentan una frecuencia mayor de uso en según qué registro. Así, por ejemplo, la palabra sitio presenta una frecuencia de uso mayor en el registro coloquial frente a la palabra lugar, la cual, a su vez, presenta una mayor frecuencia de uso en el registro formal.

Cabe añadir que nuestra intención no es únicamente mostrar si es así entre los usuarios de una L1, sino también comprobar si estas preferencias léxicas se dan entre los usuarios de una L2. Las parejas de preferencias léxicas analizadas son:

- uso de cosa (coloquial) por objeto (formal),

- uso de mejor (coloquial) por preferible (formal),

- uso de primero (coloquial) por en primer lugar (formal),

- uso de sitio (coloquial) por lugar (formal),

- uso de así (coloquial) por de este modo (formal),

- uso de casi (coloquial) por apenas (formal),

- uso de a lo mejor (coloquial) por quizá/-s (formal),

- uso de de verdad (coloquial) por verdaderamente (formal),

- uso de pero (coloquial) por aunque (formal),

- uso de raro (coloquial) por extraño (formal).

\section{CONOCIMIENTO, RECONOCIMIENTO Y PERCEPCIÓN}

A lo largo de 2018 presentamos en diferentes Congresos ${ }^{1}$ los conceptos de conocimiento, reconocimiento y percepción, los cuales consideramos pertinentes a la hora de analizar los registros tanto en una L1 así como contrastándola con una L2.

\footnotetext{
${ }^{1}$ Nos referimos al II Congreso Internacional del español hablado: XXV aniversario del grupo de investigación Val.Es.Co., celebrado en la Universidad de Valencia (España) entre los días 17 y 19 de octubre de 2018 y al VII Simposio Internacional de Hispanistas: Encuentros 2018 celebrado en la Universidad de Łódź (Polonia), entre los días 24 y 26 de mayo de 2018. Evidentemente, a lo largo de los
} 
El concepto de conocimiento nos ayuda a responder a la pregunta sobre si el aprendiz conoce el aprendiz el significado de un determinado recurso de la lengua. Evidentemente, es un factor que debe tenerse en cuenta sobre todo en el análisis de la interlengua de un aprendiz de L2. Sin embargo, también se debe considerar que, en determinadas ocasiones, podemos encontrarnos ante ciertas características del registro coloquial que no van a ser conocidas por todos los hablantes de una L1 y, por lo tanto, también puede ayudar a delimitar la influencia de otras variaciones. En el caso de nuestro estudio se trataría de comprobar si todos los elementos de las parejas analizadas son conocidos por los sujetos de estudio.

El concepto de reconocimiento responde a la pregunta sobre si puede el aprendiz determinar si un determinado recurso es propio del registro formal o del coloquial. En este caso se trata de comprobar si realmente los sujetos de estudio colocan uno de los miembros de las parejas en el ámbito de lo coloquial y el otro en el ámbito de lo formal.

Finalmente, el concepto de percepción responde a la pregunta sobre hasta qué punto puede el aprendiz precisar si un recurso es más o menos formal o más o menos coloquial. Es decir, se trataría de no solamente delimitar un recurso dentro del ámbito de lo coloquial, sino de describir la carga intrínseca de coloquialidad o formalidad que conlleva cada recurso.

\section{EL ESTUDIO}

En los siguientes apartados presentamos la metodología adoptada para la obtención de los datos y las características de los participantes del estudio.

\subsection{Metodología del estudio}

A los participantes del estudio se les presentó una ficha con un listado de veinte palabras o grupos de palabras, los cuales formaban diez parejas de preferencias léxicas. No obstante, aparecían de forma aleatoria, de manera que los participantes no pudieran deducir la vinculación que existía en unos elementos y otros.

Los participantes fueron instruidos para que puntuaran de 5 a -5 cada palabra o grupo de palabras presentadas, representando el 5 el mayor nivel de formalidad y el -5 el mayor nivel de coloquialidad. Hemos considerado las respuestas entre el 0,5 y el 0,5 como equivalentes a un valor neutro entre formalidad y coloquialidad. $\mathrm{Si}$ alguna de las palabras o grupo de palabras resultara desconocida para los participantes, debían dejar ese elemento sin puntuar.

próximos meses aparecerán las pertinentes publicaciones derivadas de dichos Congresos, así como otros artículos en los que se desarrollan los conceptos de conocimiento, reconocimiento y percepción. 
Los elementos aparecían sin contexto ni ninguna explicación que pudiera modificar la percepción que los sujetos tenían de ellos. Como bien es sabido, la intención en cuanto al uso de cualquier palabra puede ser modificada gracias a otros elementos que ayudan a atenuar o intensificar el mensaje. No obstante, nuestra intención no era comprobar si los participantes eran capaces de reconocer y percibir los diferentes mecanismos de atenuación o intensificación, etc., sino de analizar el reconocimiento y la percepción intrínseca de una palabra o grupo de palabras.

\subsection{Participantes}

Hasta la fecha en el estudio han participado 78 sujetos, todos los cuales eran estudiantes de Filología Hispánica de la Universidad Adam Mickiewicz de Poznan (Polonia), durante los cursos académicos 2015-2016, 2016-2017 y 2017-2018. Los resultados se han analizado teniendo en cuenta tres grupos de participantes:

- Estudiantes polacos acogidos al programa Erasmus. Este grupo ha estado formado por 40 estudiantes del tercer curso de los Estudios de Grado de nivel $\mathrm{C} 1$, todos los cuales habían realizado una estancia en una universidad española de un semestre de duración. Por lo tanto, su aprendizaje del español como lengua extranjera ha sido en contexto académico y natural.

- Estudiantes polacos no acogidos al programa Erasmus. Este grupo ha estado integrado por 28 estudiantes del tercer curso de los Estudios de Grado de nivel $\mathrm{C} 1$, ninguno de los cuales había visitado un país de habla española. Así, su aprendizaje del español como lengua extranjera solo ha transcurrido en contexto académico.

- Nativos. El tercer grupo, al cual tratamos como grupo de control, ha estado formado por 10 estudiantes españoles, los cuales realizaban una estancia semestral en la Universidad Adam Mickiewicz de Poznan acogidos al programa Erasmus.

No consideramos cerrado el número de participantes ni la toma de datos, ya que nuestra intención es que cada uno de los tres grupos esté constituido por un total de 50 sujetos. Sin embargo, queremos destacar la uniformidad de los datos presentados, por lo que no consideramos que varíen significativamente una vez alcanzado el número máximo de participantes previstos.

\section{ANÁLISIS DE LOS DATOS}

En los siguientes apartados presentamos los resultados obtenidos respecto a las diferentes parejas analizadas. 


\subsection{La pareja cosa/objeto}

La pareja cosa/objeto muestra un comportamiento que podríamos considerar previsible - según la definición propuesta por Gaviño Rodríguez (2008) - de las preferencias léxicas. Como se puede observar en la tabla 1, los tres grupos reconocen el elemento cosa como coloquial y el elemento objeto como formal.

Tabla 1. Reconocimiento y percepción de la pareja cosa/objeto

\begin{tabular}{|l|c|c|c|}
\cline { 2 - 4 } \multicolumn{1}{c|}{} & Sin estancia & Con estancia & Nativos \\
\hline cosa & $-1,350$ & $-1,750$ & $-2,200$ \\
\hline objeto & 0,975 & 1,429 & 1,000 \\
\hline
\end{tabular}

No obstante en la tabla 2 se muestra como la diferencia perceptual que tienen los nativos de los elementos de la pareja es mayor que la de los miembros del grupo de estudiantes polacos sin estancia en un país hispanohablante. Sin embargo, esta diferencia perceptual desaparece entre los nativos y los estudiantes polacos que han realizado una estancia Erasmus.

Tabla 2. Diferencia perceptual ${ }^{2}$ entre los elementos de la pareja cosa/objeto

\begin{tabular}{|l|c|}
\cline { 2 - 2 } \multicolumn{1}{c|}{} & cosa/objeto \\
\hline Sin estancia & 2,325 \\
\hline Con estancia & 3,179 \\
\hline Nativos & 3,2 \\
\hline
\end{tabular}

\subsection{La pareja mejor/preferible}

En el caso de la pareja mejor/preferible los tres grupos otorgan un valor neutro al primer elemento y formal al segundo; es decir, no hay un elemento que se considere coloquial, pero sí uno con una carga formal mayor.

Tabla 3. Reconocimiento y percepción de la pareja mejor/preferible

\begin{tabular}{|l|c|c|c|}
\cline { 2 - 4 } \multicolumn{1}{c|}{} & Sin estancia & Con estancia & Nativos \\
\hline mejor & $-0,025$ & 0,286 & 0,200 \\
\hline preferible & 2,000 & 2,464 & 2,000 \\
\hline
\end{tabular}

${ }^{2}$ La diferencia perceptual es la diferencia puntual que existe entre los dos elementos de una pareja. Así, si al elemento A se le otorga un valor de -1 y al elemento B un valor de 2 , la diferencia perceptual entre ambos elementos será de 3 . Evidentemente, una diferencia perceptual de 4 no representa lo mismo que una diferencia de 0,4. 
El comportamiento de los tres grupos es en este caso tan similar que incluso en la tabla 4 se muestra como la diferencia perceptual es casi inexistente.

Tabla 4. Diferencia perceptual entre los elementos de la pareja mejor/preferible

\begin{tabular}{|l|c|}
\cline { 2 - 2 } \multicolumn{1}{c|}{} & mejor/preferible \\
\hline Sin estancia & 2,025 \\
\hline Con estancia & 2,178 \\
\hline Nativos & 2,2 \\
\hline
\end{tabular}

\subsection{La pareja primero/en primer lugar}

En la presente pareja de nuevo aparece un elemento claramente reconocido como formal en los tres grupos. Sin embargo, cabe señalar que el elemento que supuestamente debería ser reconocido como coloquial aparece con valores positivos también en los tres grupos.

Se observa también una diferencia considerable en cuanto al elemento en primer lugar entre el grupo de control y los dos grupos de estudiantes polacos. Estos perciben el elemento con un mayor grado de formalidad respecto al grupo de nativos. Cabe suponer que esto es debido a que durante el aprendizaje se potencia el uso de dicho elemento en discursos claramente académicos escritos y orales.

Tabla 5. Reconocimiento y percepción de la pareja primero/en primer lugar

\begin{tabular}{|l|c|c|c|}
\cline { 2 - 4 } \multicolumn{1}{c|}{} & Sin estancia & Con estancia & Nativos \\
\hline primero & 0,350 & 0,607 & 0,600 \\
\hline en primer lugar & 2,475 & 2,857 & 1,800 \\
\hline
\end{tabular}

Como consecuencia de lo comentado en el párrafo anterior deriva que la diferencia perceptual presentada por los dos grupos de estudiantes polacos sea mucho mayor que la del grupo de control.

Tabla 6. Diferencia perceptual entre los elementos de la pareja primero/en primer lugar

\begin{tabular}{|l|c|}
\cline { 2 - 2 } \multicolumn{1}{c|}{} & primero/en primer lugar \\
\hline Sin estancia & 2,125 \\
\hline Con estancia & 2,25 \\
\hline Nativos & 1,2 \\
\hline
\end{tabular}




\subsection{La pareja sitio/lugar}

Los tres grupos de participantes presentan resultados diferentes en el caso de la pareja sitio/lugar. El grupo de control muestra unos datos previsibles según los cuales sitio es reconocido claramente como coloquial y lugar como formal. En cambio, para el grupo de estudiantes polacos que no ha realizado ninguna estancia en un país de habla española, los dos elementos están situados en el ámbito de lo neutro. Por otra parte, el grupo de estudiantes polacos que ha realizado una estancia en el marco del programa Erasmus reconoce el elemento lugar como formal, igual que el grupo de control, aunque el elemento sitio lo considera de forma neutra, como el grupo sin estancias.

Como en otros casos, que un elemento sea tenido como neutro por parte de los grupos de estudiantes polacos, mientras que por el grupo de control sea tenido como coloquial, se debe a la idea por parte del aprendiz de que los estadios iniciales del aprendizaje del español corresponden a un registro neutro en el que no aparecen elementos coloquiales.

Tabla 7. Reconocimiento y percepción de la pareja sitio/lugar

\begin{tabular}{|l|c|c|c|}
\cline { 2 - 4 } \multicolumn{1}{c|}{} & Sin estancia & Con estancia & Nativos \\
\hline sitio & $-0,275$ & 0 & $-2,600$ \\
\hline lugar & 0,275 & 0,714 & 0,800 \\
\hline
\end{tabular}

La diferencia perceptual mostrada en la tabla 8 es la consecuencia de lo mencionado anteriormente. Sin embargo, si bien en el apartado 4.3 la diferencia perceptual presentada por el grupo de control era claramente inferior a la de los grupos de estudiantes polacos, en el caso de la pareja sitio/lugar ocurre al contrario, mostrando una diferencia mucho mayor.

Tabla 8. Diferencia perceptual entre los elementos de la pareja sitio/lugar

\begin{tabular}{|l|c|}
\cline { 2 - 2 } \multicolumn{1}{c|}{} & sitio/lugar \\
\hline Sin estancia & 0,55 \\
\hline Con estancia & 0,714 \\
\hline Nativos & 3,4 \\
\hline
\end{tabular}

\subsection{La pareja así/de este modo}

La pareja asi/de este modo presenta unos datos similares a los de sitio/lugar en el sentido de que si bien reconoce claramente el primer elemento como coloquial, los grupos de estudiantes polacos lo consideran neutro. No obstante los valores en los tres grupos son negativos. 
Por otro lado, los tres grupos reconocen claramente el elemento de este modo como formal.

Tabla 9. Reconocimiento y percepción de la pareja así/de este modo

\begin{tabular}{|l|c|c|c|}
\cline { 2 - 4 } \multicolumn{1}{c|}{} & Sin estancia & Con estancia & Nativos \\
\hline así & $-0,308$ & $-0,179$ & $-1,200$ \\
\hline de este modo & 2,375 & 2,571 & 2,200 \\
\hline
\end{tabular}

En cuanto a la diferencia perceptual, la tendencia de los grupos de estudiantes polacos tiende a aproximarse a la del grupo de control tras una estancia en un país de habla española, tal y como se observa en la tabla 10.

Tabla 10. Diferencia perceptual entre los elementos de la pareja así/de este modo

\begin{tabular}{|l|c|}
\cline { 2 - 2 } \multicolumn{1}{c|}{} & así/de este modo \\
\hline Sin estancia & 2,683 \\
\hline Con estancia & 2,75 \\
\hline Nativos & 3,4 \\
\hline
\end{tabular}

\subsection{La pareja casi/apenas}

Como en los casos anteriores, uno de los elementos de la pareja analizada aparece claramente reconocido como formal en los tres grupos. El elemento casi, sin embargo, no aparece reconocido como coloquial, sino que en los tres grupos se situaría en el ámbito de lo neutro. No obstante, cabe señalar que si bien muestra valores positivos en el grupo de control, en los dos grupos de estudiantes polacos los valores son negativos.

Tabla 11. Reconocimiento y percepción de la pareja casi/apenas

\begin{tabular}{|l|c|c|c|}
\cline { 2 - 4 } \multicolumn{1}{c|}{} & Sin estancia & Con estancia & Nativos \\
\hline casi & $-0,231$ & $-0,071$ & 0,400 \\
\hline apenas & 1,538 & 1,750 & 1,600 \\
\hline
\end{tabular}

La diferencia perceptual entre los tres grupos no es destacable, como se observa en la tabla 12. Sin embargo, debemos mencionar que en los dos grupos de aprendices polacos la diferencia es mayor que la presentada por el grupo de control. Cabe 
suponer que esto se deba de nuevo al efecto del proceso de aprendizaje, puesto que el elemento apenas se introduce con posterioridad a casi, cuando el nivel de lengua se encuentra en un nivel más avanzado.

Tabla 12. Diferencia perceptual entre los elementos de la pareja casi/apenas

\begin{tabular}{|l|c|}
\cline { 2 - 2 } \multicolumn{1}{c|}{} & casi/apenas \\
\hline Sin estancia & 1,769 \\
\hline Con estancia & 1,821 \\
\hline Nativos & 1,4 \\
\hline
\end{tabular}

\subsection{La pareja a lo mejor/quizá,-s}

La pareja a lo mejor/quizá,-s supone un caso diferente a los anteriores, puesto que los datos presentados por los dos grupos de aprendices polacos son diferentes a los obtenidos por el grupo de control tanto en el elemento supuestamente coloquial como en el formal.

En el grupo de control los datos responden a lo esperado, ya que el elemento quizá,-s es reconocido claramente como formal y el elemento a lo mejor tiene valores negativos, aunque está en el ámbito de lo neutro. Sin embargo, para los dos grupos de aprendices polacos, el elemento a lo mejor es el que se sitúa en el ámbito de lo formal, mientras que el elemento quizá,-s presenta valores negativos, aunque todavía esté en el ámbito de lo neutro.

Como ya hemos comentado en apartados anteriores, es de suponer que la influencia del proceso de aprendizaje sea la causante de esta diferencia entre el grupo de control y los de aprendices polacos, ya que el elemento quizá,-s aparece en los estadios iniciales, mientras que a lo mejor se da en estadios posteriores, por lo que parece que existe por parte del aprendiz de español como lengua extranjera cierta identificación entre registros y nivel de conocimiento de una lengua.

Tabla 13. Reconocimiento y percepción de la pareja a lo mejor/quizá,-s

\begin{tabular}{|l|c|c|c|}
\cline { 2 - 4 } \multicolumn{1}{c|}{} & Sin estancia & Con estancia & Nativos \\
\hline a lo mejor & 0,750 & 0,536 & $-0,400$ \\
\hline quizá,-s & $-0,125$ & $-0,179$ & 2,000 \\
\hline
\end{tabular}

La diferencia perceptual también muestra un comportamiento diferente entre los dos grupos polacos, para los cuales los dos elementos analizados no son percibidos de manera tan opuesta como en el caso del grupo de control. 
Tabla 14. Diferencia perceptual entre los elementos de la pareja a lo mejor/quizá,-s

\begin{tabular}{|l|c|}
\cline { 2 - 2 } \multicolumn{1}{c|}{} & a lo mejor/quizá,-s \\
\hline Sin estancia & 0,875 \\
\hline Con estancia & 0,715 \\
\hline Nativos & 2,4 \\
\hline
\end{tabular}

\subsection{La pareja de verdad/verdaderamente}

A primera vista la pareja de verdad/verdaderamente muestra un comportamiento previsible, puesto que uno de los elementos aparece reconocido claramente como formal frente al otro. Sin embargo, si observamos los datos detenidamente, comprobaremos que existe una clara diferencia entre los obtenidos del grupo de control y los de los grupos de aprendices polacos.

Para los dos grupos de aprendices polacos el elemento verdaderamente es reconocido como formal, pero con una percepción del grado de formalidad mucho mayor que la presentada por el grupo de control. En cambio, el elemento de verdad es reconocido como coloquial por el grupo de control, frente a los grupos de aprendices polacos que lo sitúan en el ámbito de lo neutro. Sin embargo, cabe señalar que los tres grupos le otorgan valores negativos.

Tabla 15. Reconocimiento y percepción de la pareja de verdad/verdaderamente

\begin{tabular}{|l|c|c|c|}
\cline { 2 - 4 } \multicolumn{1}{c|}{} & Sin estancia & Con estancia & Nativos \\
\hline de verdad & $-0,450$ & $-0,179$ & $-0,800$ \\
\hline verdaderamente & 2,450 & 2,536 & 1,200 \\
\hline
\end{tabular}

En la tabla 16 se muestra cómo la diferencia perceptual de los dos grupos de aprendices polacos es mayor que la presentada por el grupo de control.

Tabla 16. Diferencia perceptual entre los elementos de la pareja de verdad/verdaderamente

\begin{tabular}{|l|c|}
\cline { 2 - 2 } \multicolumn{1}{c|}{} & de verdad/verdaderamente \\
\hline Sin estancia & 2,9 \\
\hline Con estancia & 2,715 \\
\hline Nativos & 2,0 \\
\hline
\end{tabular}




\subsection{La pareja pero/aunque}

La pareja pero/aunque también presenta un comportamiento inesperado en cuanto a los resultados del grupo de control. Según cabría esperar (Gaviño Rodríguez 2008: 76), el elemento aunque debería ser reconocido claramente como formal respecto al elemento pero. Sin embargo, ambos se sitúan en el ámbito de lo neutro con valores muy aproximados.

Son los dos grupos de aprendices polacos los que en este caso presentan los valores esperados al reconocer claramente como formal el elemento aunque. El elemento pero se sitúa en el ámbito de lo neutro, pero con valores negativos.

Tabla 17. Reconocimiento y percepción de la pareja pero/aunque

\begin{tabular}{|l|c|c|c|}
\cline { 2 - 4 } \multicolumn{1}{c|}{} & Sin estancia & Con estancia & Nativos \\
\hline pero & $-0,475$ & $-0,286$ & 0,200 \\
\hline aunque & 1,125 & 1,464 & 0,400 \\
\hline
\end{tabular}

El comportamiento de los grupos de aprendices polacos parece responder, como ya hemos visto en algunos apartados anteriores, a la influencia del proceso de aprendizaje. La conjunción aunque se introduce no en los niveles iniciales sino una vez se alcanza el nivel de referencia B1 e implica el manejo de las oraciones subordinadas y del modo subjuntivo. Así, los aprendices de nuevo parecen identificar los registros con los niveles de aprendizaje, siendo los niveles iniciales de un registro aparentemente neutro y ganándose formalidad a medida que se avanza en el conocimiento de la lengua. Aparentemente, la influencia del proceso de aprendizaje es mayor que la que haya tenido la estancia en un país hispanohablante.

La diferencia perceptual refleja lo comentado anteriormente. Por una parte, para los dos grupos de aprendices polacos la diferencia entre ambos elementos es clara. En cambio, para el grupo de control es casi inexistente.

Tabla 18. Diferencia perceptual entre los elementos de la pareja pero/aunque

\begin{tabular}{|l|c|}
\cline { 2 - 2 } \multicolumn{1}{c|}{} & pero/aunque \\
\hline Sin estancia & 1,6 \\
\hline Con estancia & 1,75 \\
\hline Nativos & 0,2 \\
\hline
\end{tabular}

\subsection{La pareja raro/extraño}

La última pareja analizada es la formada por los elementos raro/extraño. En este caso el comportamiento de los tres grupos es el esperado al reconocer uno de los elementos como claramente formal frente al otro, al cual se le asignan valores 
negativos. En todo caso, el grupo de aprendices polacos sin estancia Erasmus, aunque otorga valores positivos al elemento extraño, estos no salen del ámbito de lo neutro. Así, en esta ocasión la estancia provoca que los valores del elemento formal se aproximen, e incluso superen en el grado de percepción formal, a los del grupo de control.

Tabla 19. Reconocimiento y percepción de la pareja raro/extraño

\begin{tabular}{|l|c|c|c|}
\cline { 2 - 4 } \multicolumn{1}{c|}{} & Sin estancia & Con estancia & Nativos \\
\hline raro & $-1,075$ & $-1,357$ & $-1,400$ \\
\hline extraño & 0,150 & 0,821 & 0,600 \\
\hline
\end{tabular}

En la tabla 20 se muestra cómo la diferencia perceptual del grupo de aprendices polacos que no han realizado ninguna estancia Erasmus es mucho más distante de las cifras presentadas por el grupo de control y cómo el grupo de aprendices polacos que sí han realizado dicha estancia aumentan la diferencia perceptual.

Tabla 20. Diferencia perceptual entre los elementos de la pareja raro/extraño

\begin{tabular}{|l|c|}
\cline { 2 - 2 } \multicolumn{1}{c|}{} & raro/extraño \\
\hline Sin estancia & 1,225 \\
\hline Con estancia & 2,178 \\
\hline Nativos & 2,0 \\
\hline
\end{tabular}

\section{CONCLUSIONES}

La principal conclusión que se puede extraer de las parejas analizadas es que, efectivamente, uno de los elementos posee una carga de formalidad superior al segundo, el cual en ocasiones se sitúa en el ámbito de lo coloquial y en otros en el de lo neutro. En el caso de los nativos la única excepción la encontramos en la pareja pero/aunque, cuyos dos elementos se perciben como neutros. En el caso de los estudiantes polacos, la única excepción está en la pareja a lo mejor/quizá,-s, la cual presenta valores contrarios a los del grupo de control. Esto sin duda es debido a la relación existente entre la percepción que se tiene de los registros por parte de los alumnos de español como lengua extranjera y el proceso de aprendizaje de dicha lengua.

En este sentido cabe destacar que ciertos elementos del español no son reconocidos como coloquiales por los aprendices, puesto que los identifican con un estadio inicial del aprendizaje y no con los registros coloquial y formal. Así ocurre, 
por ejemplo, con los elementos sitio, asi y de verdad, los cuales son considerados como neutros por los aprendices polacos frente a los nativos, quienes los sitúan en el ámbito de lo coloquial.

Asimismo, otros elementos tienden a percibirse con un mayor grado de formalidad que en el caso del grupo de control debido a que durante el proceso de aprendizaje dichos elementos aparecen en niveles no iniciales. Así ocurre, por ejemplo, con los elementos en primer lugar, aunque y verdaderamente, los cuales son tenidos por los aprendices polacos con un mayor nivel de formalidad que los nativos.

En definitiva, aunque las preferencias léxicas existen como tales tanto en una L1 como en una L2, no debemos olvidar que el proceso de aprendizaje influye en la percepción que los aprendices de una L2 tienen sobre los diferentes elementos. 


\section{BIBLIOGRAFÍA}

Anusiewicz, J. (1992). Potoczność jako sposób doświadczania świata i jako postawa wobec świata. In J. Anusiewicz, F. Nieckula (eds.), Język a kultura, t. 5: Potoczność w języku i w kulturze (pp. 9-20). Wrocław: Wydawnictwo Uniwersytetu Wrocławskiego.

Bartmiński, J. (1992). Styl potoczny. In J. Anusiewicz, F. Nieckula (eds.), Język a kultura, t. 5: Potoczność $w$ języku $i$ w kulturze (pp. 37-54). Wrocław: Wydawnictwo Uniwersytetu Wrocławskiego.

Bartmiński, J. (1993). Styl potoczny. In J. Bartmiński (ed.), Encyklopedia kultury polskiej XX wieku, t. 2 (pp. 115-134). Lublin: Wiedza o Kulturze.

Briz Gómez, A. (1996). El español coloquial. Situación y uso. Madrid: Arco Libros.

Coseriu, E. (1981). Lecciones de lingüistica general. Madrid: Gredos.

Gaviño Rodríguez, V. (2008). Español coloquial. Pragmática de lo cotidiano. Cádiz: Servicio de publicaciones de la Universidad de Cádiz.

Hołowka, T. (1986). Myślenie potoczne. Heterogeniczność zdrowego rozsq̨dku. Warszawa: Państwowy Instytut Wydawniczy.

Zdunkiewicz-Jedynak, D. (2008). Wyktady ze stylistyki. Warszawa: PWN. 